\title{
Loss optimization of transverse Bragg resonance waveguides
}

\author{
John M. Choi, Wei Liang, Yong Xu, and Amnon Yariv
}

California Institute of Technology, M/S 136-93, 1200 East California Boulevard, Pasadena, California 91125

Coupled-mode theory was used to analyze guiding in a transverse Bragg resonance (TBR) waveguide structure composed of a GaAs substrate with air holes. This analysis predicts that propagation loss will be minimized for discrete widths of the waveguide core. Although the coupled-mode theory is normally applied to structures with small index perturbations, two-dimensional finite-difference time-domain simulations of the TBR waveguide show good quantitative agreement with the coupled-mode predictions, and these results corroborate the previously predicted existence of discrete core widths for low-loss propagation. (C) 2004 Optical Society of America

OCIS codes: $230.1480,230.7370,130.2790$.

\section{INTRODUCTION}

Optical waveguiding by Bragg reflection ${ }^{1-3}$ has garnered much interest as a result of recent work in planar photonic crystal waveguides. ${ }^{4-6}$ As a generalization of these photonic crystal waveguides, the transverse Bragg resonance (TBR) waveguide was recently proposed and analyzed. $^{7}$ Instead of relying on time-consuming numerical solutions to Maxwell's equations, ${ }^{8,9}$ a coupledmode formalism was applied to calculate the dispersion and loss of a TBR waveguide composed of a GaAs substrate with air holes. The results of the coupled-mode analysis predict discrete, quantized values for the width of the guiding channel for achieving low-loss TBR waveguides. Comparisons with two-dimensional finitedifference time-domain (2D FDTD) simulations indicate that the coupled-mode theory can provide a valid model of the waveguiding in a TBR waveguide even with a large index contrast and, with an empirical coupling constant, can quantitatively describe the propagation of the guided modes.

\section{COUPLED-MODE ANALYSIS}

The waveguide geometry is shown in Fig. 1. Assuming a small index pertrubation, the propagating field can be described by the Helmholtz equation,

$$
\nabla^{2} \mathbf{E}+\mu \epsilon(\mathbf{r}) \omega^{2} \mathbf{E}=0
$$

which describes the $\mathbf{E}$ field out of the plane, corresponding to a TM-like mode in a photonic crystal. Although the dielectric constant $\epsilon(\mathbf{r})$ can be complex, ${ }^{10}$ we will consider only the passive case where $\epsilon(\mathbf{r})$ is purely real. We assume a solution of the form

$$
\begin{aligned}
\mathbf{E}(\mathbf{r}, t)= & E(x) E(z, t) \\
= & {[A(x) \exp (i k x)} \\
& +B(x) \exp (-i k x)] \exp (i \omega t-i \beta z)
\end{aligned}
$$

where the propagation constant is a complex quantity, $\beta$ $=\beta_{R}+i \beta_{I}$, allowing the imaginary part of $\beta$ to account for the propagation loss due to leakage through a finite cladding. From Eqs. (1) and (2) and assuming that $\left|\beta_{I}\right|$ $\ll\left|\beta_{R}\right|$ (low loss) and $\left|\partial^{2} / \partial x^{2}\right| \ll|2 k \partial / \partial x|$ (the slowly varying approximation), we obtain the coupled-mode equations, here presented in matrix form,

$$
\begin{aligned}
\frac{\partial}{\partial x}\left(\begin{array}{l}
A(x) \\
B(x)
\end{array}\right) & =\left[\begin{array}{cc}
-(\gamma-i \Delta k) & \kappa \\
\kappa^{*} & (\gamma-i \Delta k)
\end{array}\right]\left(\begin{array}{l}
A(x) \\
B(x)
\end{array}\right) \\
\gamma & =-\frac{\beta_{R} \beta_{I}}{k}, \\
\kappa & =i \frac{k_{0}^{2}}{2 \bar{\epsilon} k} \frac{1}{a b} \int_{a b} \Delta \epsilon(\mathbf{r}) \exp (-i 2 k x) \mathrm{d} x \mathrm{~d} z, \\
\Delta k & =k_{W}-k,
\end{aligned}
$$

where $\bar{\epsilon}$ is the average of $\epsilon(\mathbf{r})$ over a unit cell of the cladding, $\Delta \epsilon(\mathbf{r}) \equiv \epsilon(\mathbf{r})-\bar{\epsilon}, k_{0}=\bar{\epsilon}^{1 / 2} \omega / c$, and, for a $2 \mathrm{D}$ triangular lattice, $k$ is defined as

$$
k= \begin{cases}k_{b}=\frac{2 \pi}{b}, & \text { cladding } \\ k_{W}=\left[\bar{\epsilon} \frac{\omega^{2}}{c^{2}}-\beta_{R}^{2}\right]^{1 / 2}, & \text { core }\end{cases}
$$

Equation (3) is solved separately in the core $(\kappa=0, \Delta k$ $=0$ ) and cladding, and the field and its derivative are required to be continuous at the interface, leading to

$$
\left(\begin{array}{l}
A_{1}(W / 2) \\
B_{1}(W / 2)
\end{array}\right)=\frac{1}{2}\left[\begin{array}{cc}
\left(1+\frac{k_{W}}{k_{b}}\right) \exp \left(i k_{W} W / 2\right) & \left.1-\frac{k_{W}}{k_{b}}\right) \exp \left(-i k_{W} W / 2\right) \\
\left(1-\frac{k_{W}}{k_{b}}\right) \exp \left(i k_{W} W / 2\right) & \left(1+\frac{k_{W}}{k_{b}}\right) \exp \left(-i k_{W} W / 2\right)
\end{array}\right]\left(\begin{array}{l}
A_{0}(W / 2) \\
B_{0}(W / 2)
\end{array}\right)
$$




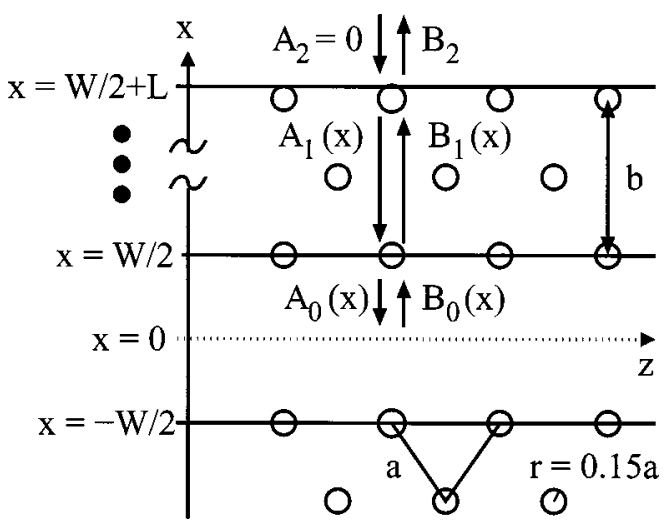

Fig. 1. TBR waveguide geometry consisting of a GaAs substrate and air holes. $x$ and $z$ are the transverse and the longitudinal dimensions, respectively, the core width is $W$, and the hole radius is $r=0.15 a$. $\quad A_{0}$ and $A_{1}$ represent the inward propagating plane-wave components in the core and cladding, respectively. $A_{2}$ represents the incoming field outside the cladding; $B_{0}, B_{1}$, and $B_{2}$ represent similar quantities for the outward-propagating components.

where $A_{1}, B_{1}$, and $A_{0}, B_{0}$ are defined by Fig. 1 . Using Eqs. (3)-(8), we can now solve for an analytic expression for the transverse field of a guided mode and consider how the waveguide design affects the loss that is due to leakage through the cladding layer.

The mirror symmetry with respect to the center of the core $(x=0)$ allows the modes to be classified by their parity: even, $A_{0}(0)=B_{0}(0)$, and odd, $A_{0}(0)$ $=-B_{0}(0)$. At the outer edge of the cladding, we observe that $A_{2}(W / 2+L)=0$ and $B_{2}(W / 2+L)$ should be minimized. Solving Eq. (3) in the cladding when $x>0$ yields a solution ${ }^{7}$ of the form

$$
\begin{aligned}
A\left(x-\frac{W}{2}\right)= & \left.-\frac{(\gamma-i \Delta k)}{S} A\left(\frac{W}{2}\right)+\frac{\kappa}{S} B\left(\frac{W}{2}\right)\right] \sinh (S x) \\
& +B\left(\frac{W}{2}\right) \cosh (S x), \\
B\left(x-\frac{W}{2}\right)= & \left.\frac{\kappa^{*}}{S} A\left(\frac{W}{2}\right)+\frac{(\gamma-i \Delta k)}{S} B\left(\frac{W}{2}\right)\right] \sinh (S x) \\
& +A\left(\frac{W}{2}\right) \cosh (S x),
\end{aligned}
$$

where $S=\left(|\kappa|^{2}+[\gamma-i \Delta k)^{2}\right]^{1 / 2}$. Applying the boundary conditions at the center of the core $\left[A_{0}(0), B_{0}(0)\right]$ and the outer edge of the cladding $\left[A_{2}(W / 2\right.$ $\left.+L), B_{2}(W / 2+L)\right]$ and requiring continuity at the core-cladding interface, Eq. (8), we solve numerically for the modal solutions. For the greatest confinement, and consequently the least loss, the transverse wave vector $k$ should be near the center of the Bragg resonance, so $k_{W}$ $\sim k_{b}$ or $\Delta k \sim 0$. Also, as $L$ increases, the leakage field $B_{2}(L+W / 2)$ becomes smaller, thus reducing the loss. A recent coupled-mode analysis of TBR waveguides ${ }^{7}$ predicts that for minimum propagation loss the width of the guiding channel (the defect) must be quantized in fractions of the transverse period. Here we examine, using an exact FDTD method, the consequences of deviating from the optimal quantized values of the width and quantify the cost in terms of increased loss resulting from such deviation. This dependence of loss on the defect width has also been observed experimentally in photonic bandgap structures. Although there are significant differences between the photonic band-gap waveguide and the TBR waveguide guided modes, these results indicate that simply removing one or more integer multiples of the periodic index variation is not optimal for low-loss waveguides.

\section{SIMULATION RESULTS}

Since a single transverse even mode is desirable for telecom applications, we study a case with a core width of $W=b / 4$. For our 2D FDTD simulations, the domain is composed of an even mirror boundary condition at $x$ $=0$, Bloch boundary conditions at $z=0$ and $z=a$, i.e., $E(z=a)=E(z=0) \exp (i \beta a)$, and an absorbing perfectly matched layer ${ }^{11}$ at the outer edge of the simulation domain as shown in Fig. 2. The cladding structure simulated was composed of approximately ten unit-cell layers. The hole radius was $r=0.15 a$, which is smaller than photonic band-gap crystals $(r \approx 0.3 a-0.4 a) .{ }^{12}$ When the core width $W$ is $b / 4$, the field envelope decays as $E(x) \sim \exp (-|\kappa| x) E_{0}$. Figure 3 shows the transverse field profile decay calculated by using the 2D FDTD simulation along with the exponentially decaying envelope

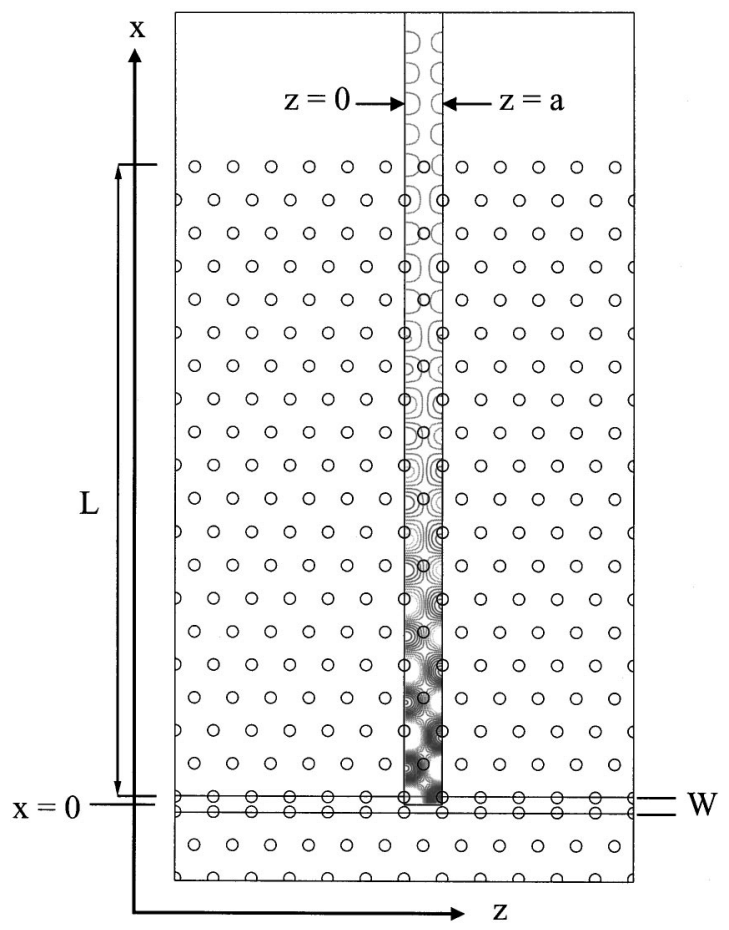

Fig. 2. 2D FDTD simulation domain showing a sample field calculation. By taking advantage of the symmetries, we reduced the domain to the slice shown in the figure. The boundary conditions were even parity at $x=0$, Bloch periodic at $z=0$ and $z=a$, and perfectly matched layers at the outermost boundary. The cladding is composed of approximately ten layers. 


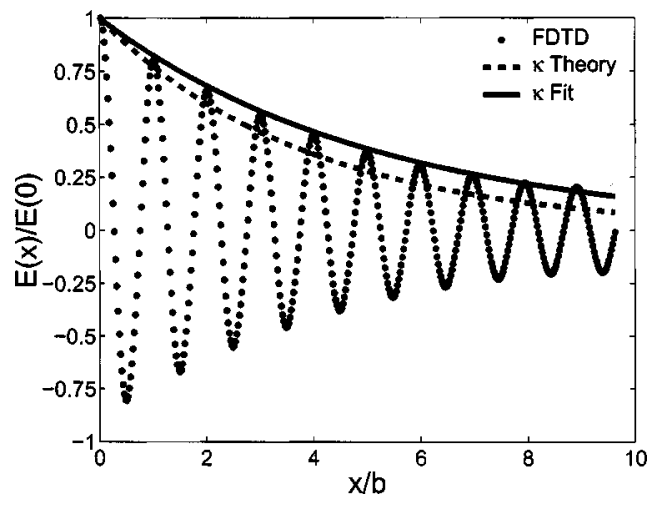

Fig. 3. Normalized transverse field decay calculated by the 2D FDTD simulation for a structure with approximately ten layers of Bragg periods. The core width $W=b / 4$. Also shown are the exponential decays predicted by the coupled-mode theory, $E(x) / E(0) \sim \exp (-|\kappa| x)$; we used the theoretical value calculated from Eq. (5), $\kappa=-i 0.1475 / a$, and an empirical fit, $\kappa$ $=-i 0.1100 / a$.

predicted by coupled-mode theory with $\kappa$ calculated from Eq. (5). Also shown is the theoretical curve with a $\kappa$ chosen to fit the FDTD results. The theoretical $\kappa$ calculated from Eq. (5) is $\kappa=-i 0.1475 / a$, and the emprical $\kappa$ found from the fit shown in Fig. 3 is $\kappa=-i 0.1100 / a$. As we shall see, this single empirical constant, and no other free parameters, will allow us to obtain nearly perfect quantitative agreement between the theory and simulations. Figure 4 shows the dispersion calculated by the coupledmode theory with use of the empirical $\kappa$ and the FDTD simulations for varying widths. Although there is very good agreement, we note that the dispersion calculated by the coupled-mode theory is not very sensitive to small changes in $\kappa$. However, the propagation loss is exponentially dependent on $\kappa$ and is a good measure of how well the coupled-mode theory agrees with the FDTD simulations.

To compare the propagation loss, we start with a $Q$ calculated by the FDTD simulation defined as

$$
Q \equiv \omega \frac{\mathcal{E}_{0}}{-\Delta P},
$$

where $\mathcal{E}_{0}$ represents the stored energy and $\Delta P=\Delta \mathcal{E} / \Delta t$ is the power dissipation. If we assume low loss and intensity decay of the form $|E(z)|^{2} \sim \exp (-\alpha z)|E(0)|^{2}$,

$$
\alpha=-2 \beta_{I}=\frac{-\Delta \mathcal{E}}{\mathcal{E}_{0}} \frac{1}{L}=\frac{\omega}{Q} \frac{\Delta t}{L}=\frac{\omega}{Q v_{g}},
$$

where we choose $L / \Delta t \equiv v_{g}=(\mathrm{d} \beta / \mathrm{d} \omega)^{-1}$. From Fig. 4 we see that the dispersion curves are qualitatively the same, meaning that the slope of the curve does not change appreciably as the width of the core varies. Thus we estimate the group velocity for all waveguides by using an analytic expression for the dispersion when the core width is $b / 4,{ }^{7}$

$v_{g} \approx\left(\frac{\mathrm{d}}{\mathrm{d} \omega} \sqrt{\frac{\bar{n}^{2}}{c^{2}} \omega^{2}-k_{b}^{2}}\right)^{-1}=\frac{c^{2} \beta}{\bar{\epsilon} \omega}=\beta \frac{c}{\bar{n}}\left(\beta^{2}+k_{b}^{2}\right)^{-1 / 2}$,

where $\bar{n}^{2}=\bar{\epsilon}$ in Eq. (5). In Fig. 5 we plot the normalized loss constant $\alpha a$ from the 2D FDTD simulations by using Eq. (12) for varying core widths with the normalized propagation constant $\beta_{R} a$ as a parameter. As predicted, ${ }^{7}$ the case $W=b / 4$ has the least propagation loss for the lowest-order even guided mode studied, and we see that the reduction in loss compared with that from a larger width can be more than an order of magnitude. In Fig. 6 the comparison of the FDTD simulations with the coupled-mode theory shows that the single empirical constant chosen from Fig. 3 is capable of fitting the data for all width parameters when $\beta_{R} a / \pi<0.6$. The large deviation from the theory near the points when 5/8 $\leqslant \beta_{R} a / \pi \leqslant 6 / 8$ is a result of the one-dimensional nature of the coupled-mode analysis. By constructing the reciprocal lattice ${ }^{13}$ for the $2 \mathrm{D}$ triangular lattice used for the cladding, as shown in Fig. 7, we see that there is a point when the forward-propagating mode $k_{1}$ can be coupled to the backward-propagating mode $k_{3}$ by the lattice vector $a_{2}$ in addition to the transverse reflection $k_{2}$, via $a_{1}$. This point results when $\beta_{R} a / \pi=2 / 3$, which corresponds to the points that show deviation from the loss expected.

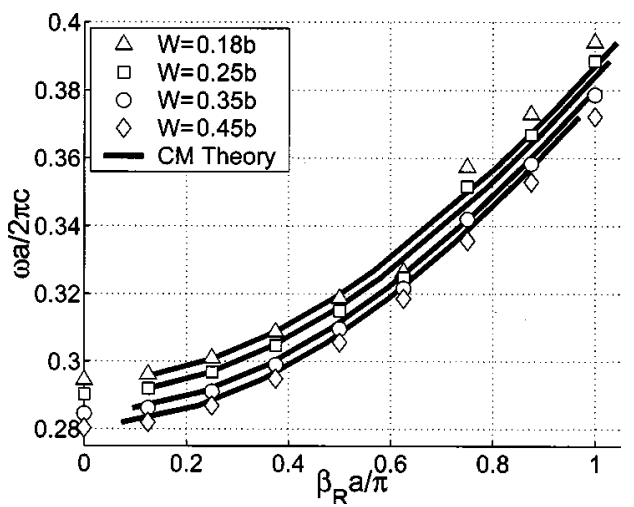

Fig. 4. Dispersion curves for varying widths of the core. Symbols, normalized frequencies from the 2D FDTD simulations; and solid curves, the corresponding dispersion curves from the coupled-mode (CM) theory in ascending order of width, $W$ $=0.18 b$ to $0.45 b$, from top to bottom.

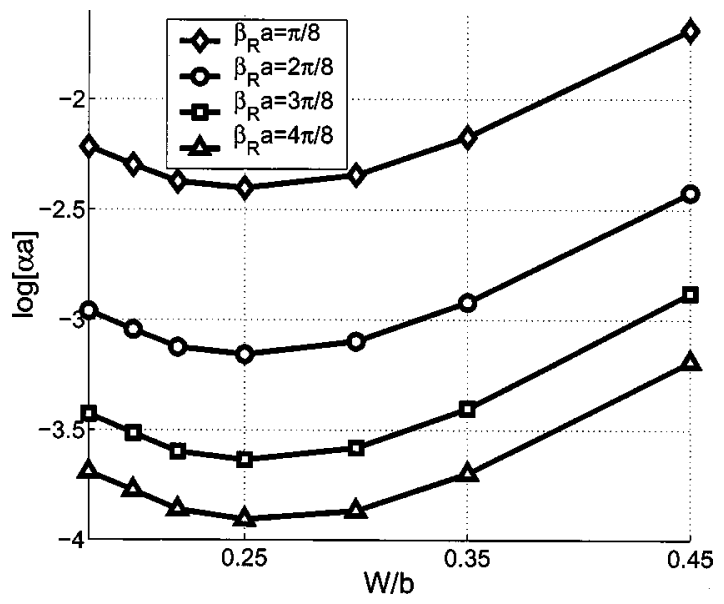

Fig. 5. Plot of normalized loss constant, $\alpha a$, from 2D FDTD simulations for varying core widths with the propagation constant $\beta_{R}$ as a parameter. For each $\beta_{R}$, a minimum in the loss is apparent when $W=b / 4$. Owing to the limits of the coupledmode theory (see Fig. 7), only the first four values of $\beta_{R}$ are shown. 


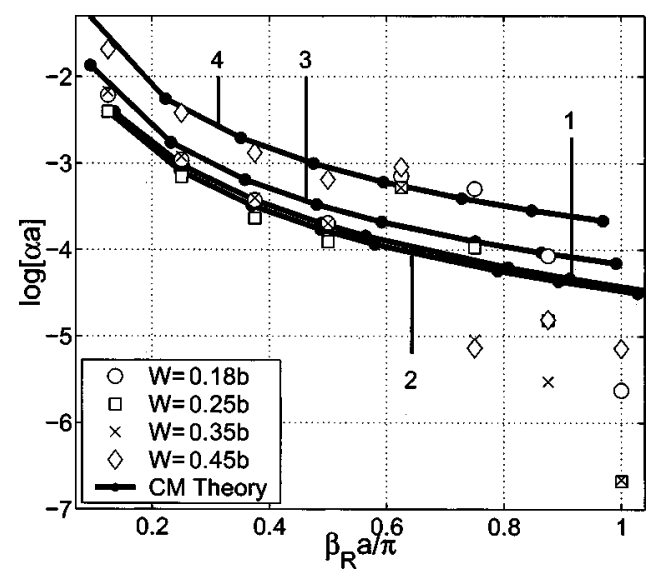

Fig. 6. Plot of the normalized loss constant from 2D FDTD simulations as well as coupled-mode (CM) theory for selected core width values. The deviation seen when $\beta_{R} a / \pi \sim 2 / 3$ is expected, as shown in Fig. 7. The solid curves corresponding to the CM theory are labeled from 1 to 4 in ascending order of width, $W=0.18 b$ to $0.45 b$.

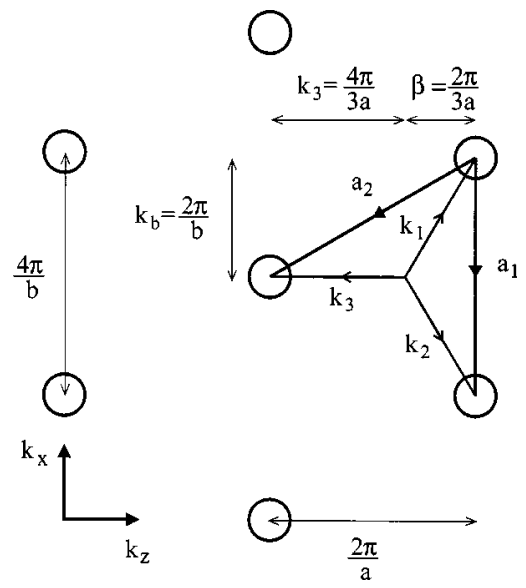

Fig. 7. Reciprocal lattice vectors for the triangular lattice, $a_{1}$ $=-4 \pi / b \hat{x}$ and $a_{2}=-2 \pi / b \hat{x}-2 \pi / a \hat{z}$. Owing to the symmetry of the triangular lattice, the coupling of $k_{1}$ to $k_{3}$ by $a_{2}$ when $\beta_{R} a / \pi \sim 2 / 3$ causes the deviation from theory seen in Fig. 6 .

Since the coupled-mode treatment was one dimensional in nature and we consider only the lowest-order Bragg reflection, it is not surprising that a $k$-vector smaller than $k_{1}$ shown in Fig. 7 is required in order for us to neglect the $2 \mathrm{D}$ periodicity of the triangular lattice cladding and obtain agreement between the analytical coupled-mode theory and the numerical FDTD simulations.

\section{CONCLUSION}

In conclusion, we have shown that it is possible to use a modified coupled-mode approach to analyze the optimal design for a low-loss transverse Bragg resonance wave- guide even for large index variations. By using an empirical coupling constant obtained through a single 2D FDTD simulation, we can characterize the periodic Bragg cladding and predict discrete core widths for minimum propagation loss that are corroborated by 2D FDTD simulations. From these simulations, we see that the coupled-mode analysis can provide quantitative predictions of the transverse field profile, the dispersion, and the propagation loss.

\section{ACKNOWLEDGMENTS}

The authors acknowledge the generous financial support of the U.S. Air Force Office of Scientific Research, the Defense Advanced Research Projects Agency, and U.S. Office of Naval Research. J. Choi (johnchoi@caltech.edu) thanks the U.S. Department of Defense and the American Society for Engineering Education for their support.

\section{REFERENCES}

1. P. Yeh and A. Yariv, "Bragg reflection waveguides," Opt. Commun. 19, 427-430 (1976).

2. A. Y. Cho, A. Yariv, and P. Yeh, "Observation of confined propagation in Bragg waveguides," Appl. Phys. Lett. 30, 471-472 (1977).

3. P. Yeh, A. Yariv, and E. Marom, "Theory of Bragg fiber," J. Opt. Soc. Am. 68, 1196-1201 (1978).

4. E. Chow, S. Y. Lin, J. R. Wendt, S. G. Johnson, and J. D. Joannopoulos, "Quantitative analysis of bending efficiency in photonic-crystal waveguide bends at $\lambda=1.55 \mu \mathrm{m}$," Opt. Lett. 26, 286-288 (2001).

5. M. Lončar, D. Nedeljkovič, T. P. Pearsall, J. Vučković, A. Scherer, S. Kuchinsky, and D. C. Allan, "Experimental and theoretical confirmation of Bloch-mode light propagation in planar photonic crystal waveguides," Appl. Phys. Lett. 80, 1689-1691 (2002).

6. M. Notomi, A. Shinya, K. Yamada, J. Takahashi, C. Takahashi, and I. Yokohama, "Structural tuning of guiding modes of line-defect waveguides of silicon-on-insulator photonic crystal slabs," IEEE J. Quantum Electron. 83, 736$742(2002)$.

7. A. Yariv, "Coupled-wave formalism for optical waveguiding by transverse Bragg reflection," Opt. Lett. 27, 936-938 (2002).

8. S. Fan, J. N. Winn, A. Devenyi, J. C. Chen, R. D. Meade, and J. D. Joannopoulos, "Guided and defect modes in periodic dielectric waveguides," J. Opt. Soc. Am. B 12, 12671272 (1995).

9. K. Sakoda, "Optical transmittance of a two-dimensional triangular photonic lattice," Phys. Rev. B 51, 4672-4675 (1995).

10. A. Yariv, Y. Xu, and S. Mookherjea, "Transverse Bragg resonance laser amplifier," Opt. Lett. 28, 176-178 (2003).

11. S. D. Gedney, "An anisotropic perfectly matched layerabsorbing medium for the truncation of FDTD lattices," IEEE Trans. Antennas Propag. 44, 1630-1639 (1996).

12. J. N. Winn, R. D. Meade, and J. D. Joannopoulos, "Twodimensional photonic band-gap materials," J. Mod. Opt. 41, 257-273 (1994).

13. N. W. Ashcroft and N. D. Mermin, Solid State Physics (Harcourt, Fort Worth, Tex., 1976). 